\title{
Author Correction: Priority list of biodiversity metrics to observe from space
}

Andrew K. Skidmore (D), Nicholas C. Coops (D), Elnaz Neinavaz D , Abebe Ali, Michael E. Schaepman (D), Marc Paganini, W. Daniel Kissling (iD, Petteri Vihervaara (D), Roshanak Darvishzadeh (D), Hannes Feilhauer (iD, Miguel Fernandez (D), Néstor Fernández (D), Noel Gorelick (D), Ilse Geijzendorffer, Uta Heiden (D), Marco Heurich (D), Donald Hobern, Stefanie Holzwarth (D), Frank E. Muller-Karger (D), Ruben Van De Kerchove, Angela Lausch (D), Pedro J. Leitão D, Marcelle C. Lock DD, Caspar A. Mücher, Brian O'Connor, Duccio Rocchini (D), Claudia Roeoesli, Woody Turner, Jan Kees Vis, Tiejun Wang (D), Martin Wegmann (D) and Vladimir Wingate

Correction to: Nature Ecology \& Evolution https://doi.org/10.1038/s41559-021-01451-x, published online 13 May 2021.

In the version of this Perspective initially published, there was an error in units reported in the main text. Specifically, in the first sentence of the sixth paragraph under the heading "A critical review of EBVs retrieved by remote sensing," in the text now reading "Finally, when harmonizing the terminology used by ecological and remote sensing communities, it is important to emphasize that utilizing broadband optical wavelengths (for example, for PlanetScope, approximately 400-700 nm) at very high spatial resolution," 400-700 nm originally appeared as "60-90 nm." The error has been corrected in the online version of the article.

Published online: 25 October 2021

https://doi.org/10.1038/s41559-021-01595-w

(C) Springer Nature Limited 2021 